\title{
Mechanical ventilation in the early phase of ST elevation myocardial infarction treated with mechanical revascularization
}

\author{
Chiara Lazzeri, Serafina Valente, Marco Chiostri, \\ Paola Attanà, Alessio Mattesini, Gian Franco Gensini \\ Intensive Cardiac Coronary Unit, Heart and Vessel Department, Azienda, \\ Ospedaliero-Universitaria Careggi, Florence, Italy
}

\begin{abstract}
Background: So far, few data have been available on the incidence and outcome of patients with acute myocardial infarction (MI) requiring mechanical ventilation (MV). The aim of the study was to assess the clinical and prognostic impact of $M V$ at short and long term in 106 patients with ST elevation MI (STEMI) requiring mechanical ventilation.

Results: The incidence of mechanical ventilation was 7.6\%. Reasons for intubation were as follows: cardiogenic shock in 64 (60.4\%) patients, ventricular fibrillation in 32 (30.1\%) patients and acute pulmonary edema in 10 (9.5\%) patients. Patients submitted to MV were older $(p=0.016)$, more frequently had a previous percutaneous coronary intervention (PCI; $p=0.014)$ and a previous MI $(p=0.001)$. A higher in-Intensive Cardiac Care Unit death was observed in MV patients (44.3\% vs. $1.5 \%, p<0.001)$, as well as a higher mortality at follow-up (36.7\% vs. $14.8 \%, p<0.001)$. MV was associated with higher mortality rates both at short and long term.

Conclusions: In a large series of consecutive STEMI patients submitted to MV, the need of $M V$ is a strong prognostic indicator of mortality both at short and long term. Among mechanically ventilated STEMI patients infarct size (as inferred by TnI values) and PCI failure were independent predictors of early death, while the duration of $M V$ was related to death at long term. (Cardiol J 2013; 20, 6: 612-617)
\end{abstract}

Key words: ST elevation myocardial infarction, mechanical ventilation, percutaneous coronary intervention, prognosis, long term

\section{Introduction}

In critically ill patients, mechanical ventilation (MV), and its impact on outcome has been extensively studied [1-3], while so far few data have been available on the incidence and outcome of patients with acute myocardial infarction (MI) requiring MV [4-7].
The present investigation was aimed at assessing the clinical characteristics and prognosis (both at short and long term) of 106 consecutive patients with ST elevation MI (STEMI) requiring $\mathrm{MV}$, all treated with primary percutaneous coronary intervention (PCI) and admitted to our Intensive Cardiac Care Unit (ICCU). 


\section{Methods}

From 1 April 2004 to 31 December 2010, 1231 consecutive patients with STEMI (within $12 \mathrm{~h}$ from symptoms' onset) [8] were admitted to our ICCU, which is located at a tertiary center. In our hospital, in Florence, the reperfusion strategy of STEMI patients is represented by primary PCI [9-12] (no exclusion criteria). STEMI patients are first evaluated by the Medical Emergency System staff in the pre-hospital setting and then directly admitted to the catheterization laboratory or transferred to it after a rapid stabilization in the First Aid. After primary PCI, they are admitted to our ICCU. Data were stored in a prospective registry on which a retrospective analysis had been carried out.

A successful procedure was defined as an infarct artery stenosis $<20 \%$ associated with Thrombolysis In Myocardial Infarction (TIMI) grade 3 flow. Failure PCI was defined as resulting in TIMI grade 0 to 2 flow, regardless the residual stenosis.

The diagnosis of STEMI was based on the criteria of the American College of Cardiology/ /American Heart Association [13]

On ICCU admission, after PCI, in a fasting blood sample the following parameters were measured: sodium $[\mathrm{mEq} / \mathrm{L}]$, glucose $[\mathrm{g} / \mathrm{L}]$, glycated hemoglobin [Hb, \%], troponin I [TnI, ng/mL], uric acid $[\mathrm{mg} / \mathrm{dL}]$, NT-pro B-type natriuretic peptide [NT-proBNP, $\mathrm{pg} / \mathrm{mL}$ ], erythrocyte sedimentation rate (ESR), leukocytes count $\left[\times 10^{3} / \mu \mathrm{L}\right]$, fibrinogen $[\mathrm{mg} / \mathrm{dL}]$ and hs-C-reactive protein positivity (hs-CRP). Creatinine $[\mathrm{mg} / \mathrm{dL}]$ was also measured, in order to calculate glomerular filtration rate [GFR, $\left.\mathrm{mL} / \mathrm{min} / 1.73 \mathrm{~m}^{2}\right]$, on admission and at discharge [14]. Glucose, TnI and creatinine were measured 3 times a day and peak values were examined. Acute insulin resistance was assessed by means of the Homeostatic Model Assessment (HOMA), as previously described [15].

Transthoracic 2-dimensional echocardiography was performed on ICCU admission in order to measure left ventricular ejection fraction (LVEF).

Ventilatory support (invasive and non-invasive ventilation), ultrafiltration (continuous-veno-venous ultrafiltration, CVVHDF) and intra-aortic balloon pump (IABP) implantation were used when needed $[9,11]$.

According to the policy of our center, sedoanalgesia (propofol + remifentanil) was administered to patients submitted to MV.

The primary endpoint was in-ICCU death and all-cause mortality at follow-up.
The study protocol was in accordance with the Declaration of Helsinki and approved by the local Ethics Committee. Informed consent was obtained from all the patients before enrollment.

\section{Statistical analysis}

Statistical analysis has been conducted with PASW 17.0 for Windows software (SPSS Inc, Chicago, IL). In all cases, a 2 -tailed $p<0.05$ has been considered statistically significant. After assessment of normality by means of Kolmogorov-Smirnov test for one sample, continuous variables have been reported as mean \pm standard deviation or median (interquartile range: IQR); categorical variables have been depicted as frequency (percentage). For continuous variables, between-groups comparisons have been performed with Student's t-test or by means of Mann-Whitney U test. Categorical variables have been compared with $\chi^{2}$ or Fisher's exact test when needed.

In the overall population predictors of death, both in-ICCU and at follow-up, have been investigated by means of logistic and Cox regression analyses, respectively. In multivariable analyses candidate variables were carefully chosen among those known to be clinically related to outcome in order to avoid model overfitting. Possible predictors for in-ICCU death were age (1 year step), admission glycemia (1 g/L step), LVEF (1\% step), admission estimated GFR (eGFR; $1 \mathrm{~mL} / \mathrm{min} / 1.73 \mathrm{~m}^{2}$ step), admission $\mathrm{Hb}(1 \mathrm{~g} / \mathrm{dL}$ step) and MV; for long-term death candidate predictors were age (1 year step), nadir eGFR $(1 \mathrm{~mL} /$ $/ \mathrm{min} / 1.73 \mathrm{~m}^{2}$ step), admission $\mathrm{Hb}(1 \mathrm{~g} / \mathrm{dL}$ step), LVEF (1\% step), previous acute MI and MV.

To adjust to the need of MV, a propensity analysis was carried out using a non-parsimonious logistic regression to determine the probability of a patient to receive MV. The variables included in the propensity score model were age, gender, previous PCI, previous MI, symptom-to-balloon time, PCI failure, length of ICCU stay, admission glycemia, peak TnI, history of hypertension, history of diabetes, smoking habit, location of the STEMI, heart rate and blood pressure at admission, and Killip's classification at admission. The goodness-of-fit of the propensity score model was obtained by the c-statistics.

\section{Results}

In our series, the incidence of MV was 7.6\% (106/1294). In the pre-hospital setting 55 patients were intubated (51.8\%), 18 patients in the Catheterization Laboratory (16.9\%), 24 patients on 
Table 1. Clinical characteristics.

\begin{tabular}{|c|c|c|c|}
\hline & $\begin{array}{c}\text { No MV } \\
\mathrm{N}=1188(91.8 \%)\end{array}$ & $\begin{array}{c}\text { MV } \\
N=106(7.6 \%)\end{array}$ & $\mathbf{P}$ \\
\hline Age [years] & $66.6 \pm 12.8$ & $69.7 \pm 13.0$ & 0.016 \\
\hline Males/females & $\begin{array}{c}883 / 305 \\
(74.3 \% / 25.7 \%)\end{array}$ & $\begin{array}{c}72 / 34 \\
(67.9 \% / 32.1 \%)\end{array}$ & 0.188 \\
\hline Body mass index $\left[\mathrm{kg} / \mathrm{m}^{2}\right]$ & $26.3 \pm 3.6$ & $25.8 \pm 3.8$ & 0.145 \\
\hline \multicolumn{4}{|l|}{ History of: } \\
\hline Diabetes mellitus & $310(24.0 \%)$ & $33(31.1 \%)$ & 0.099 \\
\hline Smoking & $812(62.8 \%)$ & $55(51.9 \%)$ & 0.027 \\
\hline COPD & $117(9.0 \%)$ & $7(6.6 \%)$ & 0.396 \\
\hline Previous $\mathrm{PCl}$ & $170(13.1 \%)$ & $23(21.7 \%)$ & 0.014 \\
\hline Previous MI & $169(13.1 \%)$ & $26(24.5 \%)$ & 0.001 \\
\hline Hypertension & $684(52.9 \%)$ & $56(52.8 \%)$ & 0.995 \\
\hline Symptoms-to-balloon time [min], median (IQR) & $226(150-300)$ & $279(180-335)$ & 0.010 \\
\hline Acute MI location: & & & 0.005 \\
\hline Anterior & $665(51.4 \%)$ & $74(69.8 \%)$ & \\
\hline Inferior & $525(40.6 \%)$ & $24(22.6 \%)$ & \\
\hline Other & $104(8.0 \%)$ & $8(7.5 \%)$ & \\
\hline Coronary artery disease: & & & 0.060 \\
\hline 1-vessel & $517(40.0 \%)$ & $30(28.3 \%)$ & \\
\hline 2-vessel & $430(33.2 \%)$ & $43(40.6 \%)$ & \\
\hline 3 - vessel & $347(26.8 \%)$ & $33(31.1 \%)$ & \\
\hline Left main coronary artery & $71(5.5 \%)$ & $14(13.2 \%)$ & 0.001 \\
\hline CABG & $31(2.4 \%)$ & $1(0.9 \%)$ & 0.508 \\
\hline $\mathrm{PCl}$ failure & $66 / 1282(5.1 \%)$ & 20/105 (19.0\%) & $<0.001$ \\
\hline Killip class: & & & $<0.001$ \\
\hline I-II & $1080(92.4 \%)$ & $42(39.6 \%)$ & \\
\hline III-IV & $89(7.6 \%)$ & $64(60.4 \%)$ & \\
\hline Admission LVEF [\%] & $42(35-50 \%)$ & $40(35-48 \%)$ & 0.025 \\
\hline LOS [h], median (IQR) & $66(48-90)$ & $96(50-168)$ & $<0.001$ \\
\hline ICCU-death & $19(1.5 \%)$ & $47(44.3 \%)$ & $<0.001$ \\
\hline Follow-up death $(n=1081)$ & $153 / 1032(14.8 \%)$ & $18 / 49(36.7 \%)$ & $<0.001$ \\
\hline
\end{tabular}

hospital admission in the Emergency Department (22.6\%) and the remaining 9 (8.7\%) patients in ICCU. Reasons for intubation were as follows: cardiogenic shock in $64(60.4 \%)$ patients, ventricular fibrillation in $32(30.1 \%)$ patients and acute pulmonary edema in $10(9.5 \%)$ patients. Non invasive ventilation was performed in 6 patients (all with acute pulmonary edema) but it was unsuccessful and they were promptly intubated. Tracheotomy was performed in 11 (10.3\%) patients, in all but one percutaneously. One patient accidentally auto-extubated and was promptly re-intubated. Among patients discharged alive, MV was stopped in 37 patients $(37 / 59,62.7 \%)$ within the first $48 \mathrm{~h}$ since ICCU admission. Ventilatory associated pneumonia was observed in $23(21.6 \%)$ patients, among whom 15 patients died.
As depicted in Table 1, patients submitted to MV were older $(p=0.016)$, more frequently had a previous $\mathrm{PCI}(\mathrm{p}=0.014)$ and a previous $\mathrm{MI}(\mathrm{p}=0.001)$. Time to symptoms-to-balloon was longer in MV patients $(\mathrm{p}=0.010)$ who showed more frequently an anterior wall MI $(\mathrm{p}=0.005)$, a higher incidence of PCI failure ( $<<0.001)$, advance Killip class $(\mathrm{p}<0.001)$ and a longer length of stay $(\mathrm{p}<0.001)$. A higher in-ICCU death was observed in MV patients $(p<0.001)$, as well as a higher mortality at follow-up.

As depicted in Table 2, MV patients showed higher values of admission and peak glycemia $(\mathrm{p}<0.001$ and $\mathrm{p}<0.001$, respectively), peak TnI $(\mathrm{p}<0.001)$, NT-proBNP $(\mathrm{p}<0.001)$, uric acid $(\mathrm{p}<0.001)$ and a higher incidence of HOMA 
Table 2. Laboratory data.

\begin{tabular}{lccc}
\hline & No MV & MV & P \\
& N = 1188 (91.8\%) & N = 106 (7.6\%) & $<0.001$ \\
\hline Admission glucose [mg/dL] & $1.30(1.09-1.60)$ & $1.82(1.35-2.71)$ & $<0.001$ \\
Peak glycemia [mg/dL] & $1.48(1.25-1.87)$ & $2.06(1.71-3.05)$ & 0.010 \\
Insulin [mU/L] & $9.5(5.6-16.8)$ & $13.8(5.2-41.8)$ & $<0.001$ \\
HOMA positivity [\%] & $106 / 757(14.0)$ & $25 / 58(53.1)$ & 0.448 \\
Glycated hemoglobin [\%] & $5.9(5.6-6.4)$ & $6.0(5.7-6.6)$ & $<0.001$ \\
Admission eGFR [mL/min/1.73 $\left.\mathrm{m}^{2}\right]$ & $85.7 \pm 30.9$ & $66.1 \pm 38.8$ & $<0.001$ \\
Nadir eGFR $\left[\mathrm{mL} / \mathrm{min} / 1.73 \mathrm{~m}^{2}\right]$ & $72.9 \pm 25.6$ & $45.2 \pm 28.3$ & 0.001 \\
Microalbuminuria $[\mathrm{mg} / \mathrm{dL}]$ & $16.7(6.3-53.9)$ & $41.6(10.6-158.0)$ & $<0.001$ \\
Peak Tnl $[\mathrm{ng} / \mathrm{mL}]$ & $80.4(35.5-171.5)$ & $128.9(59.1-384.9)$ & $<0.001$ \\
NT-proBNP $[\mathrm{pg} / \mathrm{mL}]$ & $1221(426-3002)$ & $3688(1682-14356)$ & $<0.001$ \\
Uric acid $[\mathrm{mg} / \mathrm{dL}]$ & $5.6 \pm 1.7$ & $7.0 \pm 2.5$ & 0.002 \\
ESR $[\mathrm{mm} / \mathrm{h}]$ & $22(12-40)$ & $33(17-55)$ & $<0.001$ \\
Leucocytes $\left[\times 10^{3} / \mu \mathrm{L}\right]$ & $10.7(8.7-13.4)$ & $13.4(11.1-18.7)$ & $<0.001$ \\
hs-CRP positivity $[\%]$ & $422 / 868(48.6)$ & $47 / 64(73.4)$ & 0.066 \\
Fibrinogen $[\mathrm{mg} / \mathrm{dL}]$ & $402(339-478)$ & $420(342-526)$ & \\
\hline
\end{tabular}

MV - mechanical ventilation; HOMA — homeostatic model assessment; eGFR - estimated glomerular filtration rate; Tnl — troponin I; NT-proBNP - NT-pro B-type natriuretic peptide; ESR — erythrocyte sedimentation rate; CRP — C-reactive protein

Table 3. Devices.

\begin{tabular}{lcccc}
\hline Variable & All patients & No MV & MV & P \\
\hline CVVHDF & $54(4.1 \%)$ & $25(2.1 \%)$ & $29(27.4 \%)$ & $<0.001$ \\
IABP & $298(23.1 \%)$ & $227(19.1 \%)$ & $71(67.0 \%)$ & $<0.001$ \\
2 device & $73(5.6 \%)$ & $21(1.7 \%)$ & $52(49.1 \%)$ & $<0.001$ \\
3 device & $31(2.3 \%)$ & IABP, CVVHDF or cPAP & MV and (IABP or CVVHDF) & $<0.001$ \\
& & 7 (ABP, CVVHDF or cPAP & MV and (IABP + CVVHDF) & $24(22.6 \%)$ \\
\hline
\end{tabular}

$\mathrm{MV}$ - mechanical ventilation; CVVHDF — continuous-venous-venous ultrafiltration; IABP — intra-aortic balloon pump; cPAP — continuous positive airway pressure ventilation

positivity ( $<<0.001)$. Lower values on admission and nadir eGFR $(\mathrm{p}<0.001$ and $\mathrm{p}<0.001$, respectively) were observed in MV patients who exhibited a higher inflammatory activation, as inferred by higher values of ESR $(p=0.02)$, leukocytes $(\mathrm{p}<0.01)$ and hs-CRP positivity $(\mathrm{p}<0.001)$.

As shown in Table 3, MV patients were more frequently submitted to CVVHDF ( $\mathrm{p}<0.001$ ) and IABP ( $<<0.001)$, as well as to 2 or 3 devices simultaneously $(\mathrm{p}<0.001)$.

At multivariable regression analysis, the following variables were independent predictors of in-ICCU death (when adjusted for admission eGFR): age (1 year step) (OR: 1.06, 95\% CI $1.03-1.10, \mathrm{p}<0.001)$; admission glycemia (1 g/L step) (OR: $1.65,95 \%$ CI 1.09-2.50, $\mathrm{p}=0.018)$; LVEF (1\% step) (OR: $0.96,95 \% \mathrm{CI}$ $0.93-1.00, \mathrm{p}=0.037$ ); MV (OR: 6.72, 95\% CI
2.93-15.38, $\mathrm{p}<0.001)$. Hosmer-Lemeshow goodness-of-fit test $\chi^{2} 11.3, \mathrm{p}=0.183$; Nagelkerke pseudo $\mathrm{R}^{2} 0.44$.

Patients were followed-up for $37.9\left(25^{\text {th }}-75^{\text {th }}\right.$ percentile: $14.5-60.7)$ months.

At multivariable Cox regression analysis, the following variables were independent predictors of death at follow-up: age (1 year step) (HR: 1.06, 95\% CI 1.05-1.08, p < 0.001); nadir eGFR $(1 \mathrm{~mL} /$ $/ \mathrm{min} / 1.73 \mathrm{~m}^{2}$ step) (HR: $0.98,95 \%$ CI $0.97-1.00$, p < 0.001); LVEF (1\% step) (HR: 0.98, 95\% CI $0.97-1.00, \mathrm{p}=0.010)$; MV (OR: $1.81,95 \% \mathrm{CI}$ $1.08-3.05, \mathrm{p}=0.026)$.

\section{Propensity score analysis}

Area under the receiving operator characteristic (ROC) curve was 0.95 (95\% CI 0.91-0.98), $\mathrm{p}<0.001$. 
According to the propensity score analysis the incidence of in-ICCU mortality was higher in patients submitted to mechanical ventilation in respect to those who were not $(44.3 \%$ vs. $12.3 \%$, $\mathrm{p}<0.001)$. At long term, the incidence of death was higher in patients who were submitted to MV, though it did not reach statistical significance (36.7 vs. $27.8, \mathrm{p}=0.292$ ).

At multivariable regression analysis MV was an independent predictor of early death (OR: 6.72, 95\% CI 2.93-15.38, $\mathrm{p}<0.001$ ).

At Cox regression analysis the following variables were independent predictors of long term mortality (when adjusted for nadir eGFR): age (1 year step) (HR: 1.08, 95\% CI 1.05-1.13, p < 0.001); MV (HR: 1.93, 95\% CI 1.02-3.65, $\mathrm{p}=0.044$ ).

\section{Among STEMI patients submitted to mechanical ventilation}

At multivariable regression analysis, the following variables were independent predictors of in-ICCU death (when adjusted for age): TnI (10 g/ $/ \mathrm{mL}$ ) (OR: 1.03, 95\% CI 1.01-1.06, $\mathrm{p}=0.003$ ); PCI failure (OR: 5.97, 95\% CI 1.76-20.23, $\mathrm{p}=0.004$ ).

At multivariable Cox regression analysis, the following variables were independent predictors of death at follow-up (when adjusted for discharge LVEF): age (1 year step) (HR: 1.07, 95\% CI $1.02-1.12, \mathrm{p}=0.005)$; duration of MV ( $1 \mathrm{~h} / \mathrm{step})$ (HR: $1.01,95 \%$ CI 1.00-1.02, $\mathrm{p}=0.001$ ).

\section{Discussion}

The main findings of the present investigation, performed in 106 consecutive STEMI patients requiring MV submitted to $\mathrm{PCI}$, are as follows: a) MV in the early phase of STEMI is not uncommon, being detectable in the $7.6 \%$ of the entire population; b) MV patients are older and show a larger infarct size (as indicated by higher values of peak TnI and NT-proBNP), a higher inflammatory activation (as inferred by higher values of hs-CRP positivity and leukocyte count); c) MV in the early phase of STEMI is associated with an increased risk of death both at short and long term.

So far, few data have been available on the prognostic impact of MV in acute MI and results are scarcely comparable due to differences in patients' selection criteria (NSTEMI vs. STEMI) $[4,6,16]$ and type of revascularization (thrombolysis vs. mechanical revascularization) $[4,7]$.

We reported, for the first time, the prognostic impact of MV in a large series of consecutive STEMI patients submitted to PCI. In our series the incidence of MV was higher in respect to that reported previously (7.6\% vs. 1.6\%) [6], but differently from Eran et al. [6] we only included STEMI patients (instead of acute coronary syndrome patients).

In accordance with previous studies $[4-6,17]$ we observed that MV patients exhibited a higher mortality rate (44.3\%). In 2001 Lopez Messa et al. [17] reported a $65.7 \%$ mortality rate among 333 mechanically ventilated patients with acute $\mathrm{MI}$ in a retrospective analysis from the Spanish registry ARIAM (Analisis Retraso Infarto Agudo Miocardio). In 157 consecutive patients with acute MI requiring MV admitted to an intensive care unit and submitted to coronary reperfusion within $12 \mathrm{~h}$ of symptom onset in-hospital mortality rate was $50.9 \%$. Similarly, in a subgroup of consecutive STEMI and NSTEMI patients of the German BEAT registry the overall hospital mortality rate was $48 \%$ [4] and in a small series of 51 consecutive acute MI patients who received $\mathrm{MV}$ for $>24 \mathrm{~h}$, mortality rate was $43 \%$. In a recent paper by Bhave et al. [5], MV was one of the predictors of the development of left ventricular dysfunction in the course of STEMI.

In our series MV is a strong independent predictor for mortality, since MV patients are older, with a larger infarct size (as indicated by peak TnI) [18-20], hemodynamic impairment (as inferred by advanced Killip class) and a higher incidence of multi-organ dysfunction (as indicated by lower eGFR and a higher use of devices, often simultaneously implanted).

Moreover, in our population, $\mathrm{MV}$ was associated with higher mortality rates even at follow-up. This is in accordance with previous findings by Zahger et al. [16] who described in 2005 a 30-day mortality of $29 \%$ and a 1-year mortality of $46 \%$ in a retrospective cohort of 267 consecutive patients admitted to the coronary care unit for an acute coronary syndrome requiring MV.

According to our data, among STEMI patients submitted to MV, infarct size (as inferred by TnI values) and PCI failure are independent predictors of early death, thus underscoring, even in this subgroup of STEMI patients, the impact on prognosis of a successful mechanical revascularization.

We observed, for the first time, that the duration of MV is related to death at long term in STEMI patients mechanically ventilated; this finding strongly suggests that MV should be withheld as soon as possible in these patients. However, further studies performed in larger cohorts are needed to confirm these data. 


\section{Conclusions}

According to our data, among STEMI patients submitted to MV, infarct size (as inferred by TnI values) and PCI failure are independent predictors of early death, thus underscoring, even in this subgroup of STEMI patients, the impact on prognosis of a successful mechanical revascularization.

We observed, for the first time, that the duration of MV is related to death at long term in STEMI patients mechanically ventilated; this finding strongly suggest that MV should be withheld as soon as possible in these patients. However further studies performed in larger cohorts are needed to confirm these data.

\section{Conflict of interest: none declared}

\section{References}

1. Carson SS, Kahn JM, Hough CL et al. ProVent Investigators: A multicenter mortality prediction model for patients receiving prolonged mechanical ventilation. Crit Care Med, 2012; 40: 1171-1176.

2. Esteban A, Anzueto A, Frutos F et al. Characteristics and outcomes in adult patients receiving mechanical ventilation. JAMA, 2002; 287: 345-355.

3. Bhattacharya B, Prashant A, Vishwanath P et al. Prediction of outcome and prognosis of patients on mechanical ventilation using body mass index, SOFA score, C-Reactive protein, and serum albumin. Indian J Crit Care Med, 2011; 15: 82-87.

4. Kouraki K, Schneider S, Uebis R et al. Characteristics and clinical outcome of 458 patients with acute myocardial infarction requiring mechanical ventilation. Results of the BEAT registry of the ALKK-study group. Clin Res Cardiol, 2011; 100: 235-239.

5. Bhave PD, Hoffmayer KS, Armstrong EJ et al. Predictors of depressed left ventricular function in patients presenting with STelevation myocardial infarction. Am J Cardiol, 2012; 109: 327-331.

6. Eran O, Novack V, Gilutz H, Zahger D. Comparison of thrombolysis in myocardial infarction, Global Registry of Acute Coronary Events, and Acute Physiology and Chronic Health Evaluation II risk scores in patients with acute myocardial infarction who require mechanical ventilation for more than 24 hours. Am J Cardiol, 2011; 107: 343-346.

7. Lesage A, Ramakers M, Daubin C et al. Complicated acute myocardial infarction requiring mechanical ventilation in the intensive care unit: prognostic factors of clinical outcome in a series of 157 patients. Crit Care Med, 2004; 32: 100-105.

8. Wijns W, Kolh P, Danchin N et al.; European Association for Percutaneous Cardiovascular Interventions. Guidelines on myocardial revascularization: The Task Force on Myocardial Reva- scularization of the European Society of Cardiology (ESC) and the European Association for Cardio-Thoracic Surgery (EACTS). Eur Heart J, 2010; 31: 2501-2555.

9. Valente S, Lazzeri C, Vecchio S et al. Predictors of in-hospital mortality after percutaneous coronary intervention for cardiogenic shock. Int J Cardiol, 2007; 114: 176-182.

10. Lazzeri C, Sori A, Chiostri M, Gensini GF, Valente S. Prognostic role of insulin resistance as assessed by homeostatic model assessment index in the acute phase of myocardial infarction in nondiabetic patients submitted to percutaneous coronary intervention. Eur J Anaesthesiol, 2009; 26: 856-862.

11. Valente S, Lazzeri C, Chiostri $M$ et al. Gender-related difference in ST-elevation myocardial infarction treated with primary angioplasty: a single-centre 6-year registry. Eur J Prev Cardiol, 2012; 19: 233-240.

12. Valente S, Lazzeri C, Chiostri M et al. Time of onset and outcome of cardiogenic shock in acute coronary syndromes. J Cardiovasc Med (Hagerstown), 2008; 9: 1235-1240.

13. Kushner FG, Hand M, Smith SC Jr et al.; 2009 focused updates: ACC/AHA guidelines for the management of patients with STelevation myocardial infarction (updating the 2004 guideline and 2007 focused update) and ACC/AHA/SCAI guidelines on percutaneous coronary intervention (updating the 2005 guideline and 2007 focused update) a report of the American College of Cardiology Foundation/American Heart Association Task Force on Practice Guidelines. J Am Coll Cardiol, 2009; 54: 2205-2241.

14. Levey AS, Stevens LA, Schmid CH et al.; CKD-EPI (Chronic Kidney Disease Epidemiology Collaboration). A new equation to estimate glomerular filtration rate. Ann Intern Med, 2009; 150: 604-612.

15. Lazzeri C, Valente S, Chiostri M, Picariello C, Gensini GF. Correlates of acute insulin resistance in the early phase of non-diabetic ST-elevation myocardial infarction. Diab Vasc Dis Res, 2011; 8: 35-42.

16. Zahger D, Maimon N, Novack V et al. Clinical characteristics and prognostic factors in patients with complicated acute coronary syndromes requiring prolonged mechanical ventilation. Am J Cardiol, 2005; 96: 1644-1648.

17. Lopez Messa JB, Andres de Llano JM, Berrocal de la Fuente CA et al.; por el Grupo ARIAM (Analisis Retraso Infarto Agudo Miocardio). Patient characteristics of acute myocardial infarction in mechanical ventilation. Data from Spanish registry ARIAM. Rev Esp Cardiol, 2001; 54: 851-859.

18. Byrne RA, Ndrepepa G, Braun S et al. Peak cardiac troponin-T level, scintigraphic myocardial infarct size and one-year prognosis in patients undergoing primary percutaneous coronary intervention for acute myocardial infarction. Am J Cardiol, 2010; 106: 1212-1217.

19. Suárez-Barrientos A, López-Romero P, Vivas D et al. Circadian variations of infarct size in acute myocardial infarction. Heart, 2011; 97: 970-976.

20. Klug G, Mayr A, Mair J et al. Role of biomarkers in assessment of early infarct size after successful p-PCI for STEMI. Clin Res Cardiol, 2011; 100: 501-510. 